\title{
Income Allocation Model between the Third-Party Trading Platform and Digital Content Providers
}

\author{
Peiyi Song, Dandan Liu \\ School of Economics and Management, Communication University of China Beijing P.R. China \\ Shuhua Cao \\ Audio-visual Archive of CCTV P.R. China
}

\begin{abstract}
The third-party Trading Platform connect providers can connect digital Contents Providers with the Demanders, providing them with information and transaction services and get the corresponding benefits, but how to determine the pattern of income distribution between the Trading Platform and digital content providers is the key for the survival and development of the Trading Platform. This article discusses the income allocation models between the Trading Platform and the digital Contents Providers under the conditions of symmetric information or asymmetric information, and concludes four principles for the development of Trading Platform, which are reasonable risk-sharing principle, balanced distribution of profits principle, group interests optimization principle, and mutual negotiation principle.
\end{abstract}

KEYWORD: The Trading Platform; Digital Content Products Providers; Allocation Model; Principle

\section{INTRODUCTION}

In the value chain of media-content industry that featured by video and audio programs, upstream enterprises are Content Providers who own content property, and downstream enterprises, including film and television production companies are performed as Content Demanders, while the Third-Party Trading Platform plays the role of information communication and value conversion. Currently in China the content industry market is lack of the trading platform for digital content products, nor the formation of a complete operating system, so it is necessary to investigate the mode of distribution of benefits among trading platforms and content providers from the theoretical level.

The market structure of Digital Media Content Product Platform could be abstracted as Figure 1. It is a third-party Trading Platform providing access services for Content Providers and Content Demanders, and plays an important role on providing information service and helping to promote transactions between both sides. The aim of the Trading Platform is to attract more users from both sides, decrease the searching costs, and make contact with each other at a relatively larger range.

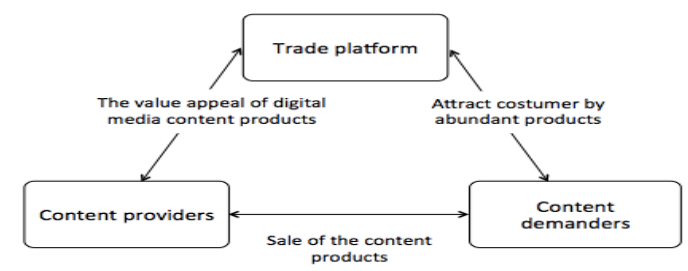

Figure 1. The basic market structure of the Trading Platform on digital content products
Finally, provide conditions and services for the transactions between both sides, and obtain profit and value.

In this market structure, Content Providers and Content Demanders are the main players in the market, while the Trading Platform, as the leading role with advantages of technology and behavior initiative, is the significant power in gaining profits. Thus, it shall firstly deal with the income allocation model between the Trading Platform and the upstream content producers or providers, which is the key factor for the healthy and steady development of the content industry chain. We construct the income allocation model under the condition of symmetric information and asymmetric information, and conclude the basic principles for the construction of the Trading Platform.

\section{INCOME ALLOCATION MODEL UNDER THE CONDITION OF SYMMETRIC INFORMATION}

Under symmetric information, the Trading Platform could observe the level of efforts made by Content Providers. Content Providers hand over various kinds of programs with property rights to the Trading Platform, and the platform gain profits by providing corresponding content services for the downstream clients.

$\pi$ represents for the total income, it relates with the investment and market circumstances 
$f$ represents the probability of an incident, that is the probability of the total income ' $\pi$ ' of the platform.

$s(\pi)$ represents the profit gained by the Content Providers, assuming both sides sign. That is $s(\pi)=s_{0}+b^{*} \pi$, and $s_{0}$ is the fixed payment.

$a$ represents for the observable effort given by the Content Providers, for instance, the sophisticated degree of content, price advantage, brand influence, etc.

$c$ represents for the observable cost paid by the Content Providers, the length of the content, for instance.

$v$ represents for the utility brought by profit gained by the Trading Platform.

$u$ represents for the utility brought by the profit gained by the Content Providers. $v$ and $u$ is the monotone increasing function of the profit.

$\bar{u}$ represents for the reservation utility, that is the utility bought by the profit if the Content Providers don't offer the content to the Trading Platform.

Under the condition of symmetric information, income allocation model is: to make the Trading Platform achieve the self-expected utility maximization.

$$
\begin{aligned}
& \max _{s(\pi)} \int v[\pi-s(\pi)] f(\pi, a) d \pi \\
& \text { s.t } \quad \int u[s(\pi)-c] f(\pi, a) d \pi \geq \bar{u}
\end{aligned}
$$

The Lagrange function is constructed,

$$
\begin{aligned}
L(s(\pi)) & =\int v[\pi-s(\pi)] f(\pi, a) d \pi \\
& +\lambda\left\{\int u[s(\pi)-c] f(\pi, a) d \pi-\bar{u}\right\}
\end{aligned}
$$

For derivation, and make it a zero, and the result is:

$$
\begin{aligned}
L^{\prime}(s(\pi)) & =(-1) v^{\prime}[\pi-s(\pi)] f(\pi, a) \\
& +u^{\prime}[s(\pi)-c] f(\pi, a)=0
\end{aligned}
$$

The first-order optimization condition is:

$$
\frac{v^{\prime}\left(\pi-s^{*}(\pi)\right)}{u^{\prime}(s(\pi)-c)}=\lambda
$$

So here is the both-sides utility formula in different profit circumstances:

$$
\frac{v^{\prime}\left(\pi-s^{*}\left(\pi_{1}\right)\right)}{v^{\prime}\left(\pi-s^{*}\left(\pi_{2}\right)\right)}=\frac{u^{\prime}\left(s\left(\pi_{1}\right)\right)}{u^{\prime}\left(s\left(\pi_{2}\right)\right)}
$$

The marginal rate of substitution is the same between the Trading Platform and Content Providers under different circumstances. This is the typical Pareto Optimality.

From above, we have the first conclusion: Pareto optimality principle: under the condition of symmetric information, Trading Platform and Content Providers have the same marginal rate of substitution in different state.
In particular, if the risk of the platform is neutral, $v$ is a constant, and $u^{\prime}\left(s\left(\pi_{1}\right)\right)=u^{\prime}\left(s\left(\pi_{2}\right)\right)$, and we have $s^{*}\left(\pi_{1}\right)=s^{*}\left(\pi_{2}\right)$, it means the best income allocation model is:

Content Providers will get the same income in all circumstances, and the platform undertakes all the risks.

\section{THE INCOME ALLOCATION MODEL UNDER THE CONDITION OF ASYMMETRIC INFORMATION}

Normally speaking, the Trading Platform usually can't observe the behavior of the Content Providers, and that is information asymmetry. Content Providers' behavior could be classified into two categories: one is the observable behavior, which is determined by the capability (recourses), and not the effort. The other is the unobservable behavior, which is determined by the effort given by the Content Providers.

Since the major task of Trading Platform is to integrate the content resources, and find the downstream clients, provide the copyright trade services, and maximize profit, and it is open to the relative cooperators in the industrial value chain, the Trading Platform's behavior heavily depends on the subjective effort. The Trading Platform's cost, like the platform construction, instrument storage, website maintenance, human resources, which are fixed investment, and it will not increase with per trade, so for the Content Providers, the unpredictability of behavior of the Trading Platform is larger. In order to calculate conveniently, we assume that all the cost of the Trading Platform was caused by the unobservable behavior.

\subsection{Basic Model}

We assume that $a_{1}, a_{2}$, relatively represents for the effort of Trading Platform and Content Providers, and cost and effort has the proportional relationship, and effort could substitute the utility brought by the cost of the enterprise.

The cost of the Trading Platform, that is the unobservable cost $c_{1}\left(a_{1}\right)$

The cost of the Trading Platform consists of two parts: effort determine the unobservable cost $c_{2}\left(a_{2}\right)$ and the observable behavior determine the cost $c_{0}$, and $c_{1}>0, c_{1}>0, c_{2}>0, c_{2}>0$; We assume $\theta$ is the exogenous variable, which is irrelevant to the effort of the enterprise, and represents for the all conditioned market circumstances.

$f$ is the probability distribution function

Level of effort chosen by the Trading Platform and Content Providers are $a_{1}, a_{2}$, the exogenous variable $\theta$ realizes, they work together to determine the total income $\pi\left(a_{1}, a_{2}, c_{0}, \theta\right)$. 
$\pi$ is the $\theta$ s strictly increasing function(relatively higher $\theta$ represents for the advantageous natural conditions), $\pi$ is $a_{1}, a_{2}$ `s strictly concave function.

Trading Platform and Content Providers will still sign a linear commission contract: $s=s_{0}+b * \pi$, among which, $s_{0}$ is the fixed income.

The profit gained by the platform is: $R=\pi-s(\pi)$ $c_{1}\left(a_{1}\right)$, at this time, the utility function of the Trading Platform is $v(R)$, and $v^{\prime}>0, v^{\prime \prime}<0$;

The profit of cooperative enterprise is $W=s(\pi)$ $c_{2}\left(a_{2}\right)-c_{0}$, and the utility function is $u(W)$, and $u>0$, $u "<0$.

The idea of the income allocation model under the condition of asymmetric information is:

Regard the maximization of expected utility of the Trading Platform as the target function of the income allocation model, and take the maximization of expected utility of the Content Providers as the constraint conditions.

The constraint conditions include two parts: First, every enterprise expects to increase its own utility through cooperation, and no enterprise wants to cooperate to decrease its own utility. So, the expected utility of registered Content Providers in the Trading Platform should be larger than the reservation utility. Set the income gained by the nonparticipating Content Provider is $W_{n}$, and the relative reservation utility is $u\left(W_{n}\right)$; Second, under any income allocation mechanism, Content Providers will choose to gain the expected utility maximization through own effort.

And the income allocation model can be showed as:

$$
\begin{aligned}
& \max _{s(\pi)} \int v(R) f\left(\pi, a_{1}, a_{2}\right) d \pi \\
& \text { s.t } \quad(I R) \quad \int u(W) f\left(\pi, a_{1}, \alpha_{2}\right) d \pi \geq u\left(W_{0}\right)
\end{aligned}
$$

(IC) get $a_{2}$, and make the $\int u(W) f\left(\pi, a_{1}, a_{2}\right) d \pi$ maximize.

Substituting the effort and cost:

$$
\begin{aligned}
& \max _{s(\pi)} \int \nu\left[\pi\left(\alpha_{1}, \alpha_{2}, c_{0}, \theta\right)-s(\pi)-c_{1}\left(\alpha_{1}\right)\right] f\left(\pi, a_{1}, a_{2}\right) d \pi \\
& \text { s.t }(I R) \quad \int u\left[s(\pi)-c_{2}\left(\alpha_{2}\right)-c_{0}\right] f\left(\pi, a_{1}, \alpha_{2}\right) d \pi \geq u\left(W_{0}\right)(1)
\end{aligned}
$$

(IC) get $a_{2}$, and make the $\int u\left[s\left(\pi-c_{2}\left(a_{2}\right)-c_{0}\right)\right] f\left(\pi, a_{1}, a_{2}\right)$ maximize.

\subsection{Model Analysis and Conclusion}

IC constrain is the problem of maximization, we could use first-order condition of this problem to substitute conditions (IC).

IC constrain is also a problem of maximization,

$$
\begin{aligned}
& \int\left\{u\left[s(\pi)-c_{2}\left(\alpha_{2}\right)-c_{0}\right] f_{\alpha_{2}}^{\prime}\left(\pi, a_{1}, \alpha_{2}\right)+\right. \\
& \left.u^{\prime}\left[s(\pi)-c_{2}\left(\alpha_{2}\right)-c_{0}\right](-1) c_{2}^{\prime}\left(\alpha_{2}\right) f\left(\pi, a_{1}, \alpha_{2}\right)\right\} d \pi=0
\end{aligned}
$$

use it to substitute (IC) condition.

Solving (1), construct Lagrange function:

$$
\begin{aligned}
& L(s(\pi))=\int v\left[\pi\left(a_{1}, \alpha_{2}, c_{0}, \theta\right)-s(\pi)-c_{1}\left(\alpha_{1}\right)\right] f\left(\pi, a_{1}, \alpha_{2}\right) d \pi+ \\
& \lambda\left\{\int u\left[s(\pi)-c_{2}\left(\alpha_{2}\right)-c_{0}\right] f\left(\pi, a_{1}, \alpha_{2}\right) d \pi-u\left(W_{0}\right)\right\}+ \\
& \mu\left\{\int \left\{u\left[s(\pi)-c_{2}\left(\alpha_{2}\right)-c_{0}\right] f_{\alpha_{2}}^{\prime}\left(\pi, a_{1}, \alpha_{2}\right)+\right.\right. \\
&\left.u^{\prime}\left[s(\pi)-c_{2}\left(\alpha_{2}\right)-c_{0}\right](-1) c_{2}^{\prime}\left(\alpha_{2}\right) f\left(\pi, a_{1}, \alpha_{2}\right) d \pi\right\}
\end{aligned}
$$

Derivative, and make it zero, get:

$$
\begin{aligned}
L^{\prime}(s(\pi)) & =v^{\prime}\left[\pi-s(\pi)-c_{1}\left(\alpha_{1}\right)\right](-1) f\left(\pi, a_{1}, \alpha_{2}\right)+ \\
& \lambda\left\{u^{\prime}\left[s(\pi)-c_{2}\left(\alpha_{2}\right)-c_{0}\right] f\left(\pi, a_{1}, \alpha_{2}\right)\right\}+ \\
& \mu\left\{u^{\prime}\left[s(\pi)-c_{2}\left(\alpha_{2}\right)-c_{0}\right] f_{\alpha_{2}}^{\prime}\left(\pi, a_{1}, \alpha_{2}\right)+\right. \\
& \left.u^{\prime \prime}\left[s(\pi)-c_{2}\left(\alpha_{2}\right)-c_{0}\right](-1) c_{2}^{\prime}\left(\alpha_{2}\right) f\left(\pi, a_{1}, \alpha_{2}\right)\right\}=0
\end{aligned}
$$

We have $\lambda^{*} \geq 0, u^{*} \geq 0$, and make the first-order condition found:

$$
\frac{v^{\prime}\left(\pi-s-c_{1}\right)}{u^{\prime}\left(s-c_{2}-c_{0}\right)}=\lambda^{*}+\mu^{*}\left[\frac{u f_{a 2}^{\prime}\left(\pi, a_{1}, a_{2}\right)}{\mu^{\prime}\left(\pi, a_{1}, a_{2}\right)}-u c_{2}^{\prime}\right]
$$

Conclusion two: under the condition of asymmetric information, the best contract can't lead to the risk configuration of the Pareto optimality and the Trading Platform could possibly deduce Content Providers to make more effort than the Pareto efficient level.

The level of the effort from Content Providers deviating from Pareto efficient level has the proportional relationship with the effort of the enterprise $a_{2}$, that is to say the more unobservable of Content Provider's effort is, and more the Trading Platform tends to deduce it to scarify more effort.

Generally speaking, the platform is more willing to take more risks than the Content Providers. Assume that the risk of platform is neutral, which means the expected utility equaling to the expected profit, without risk cost. While risk of the content provider is avoidant, the risk in the profit will bring enterprise extra risk cost. Assume that the risk avoidance coefficient is $k(k>0)$ and add VAR(value at risk), which means under certain confidence level, the biggest possible loss of some financial asset in a period of time. And the risk cost is:

$c=\frac{1}{2} k * \operatorname{Var}(s)=\frac{1}{2} k b^{2} \delta^{2}$

So the equivalent form of model in (1)

$$
\begin{aligned}
& \max _{s(\pi)}(1-b)(\pi)-c_{1}\left(\alpha_{1}-s_{0}\right) \\
& \text { s.t.(IR) } \quad s_{0}+b(\tau)-\frac{1}{2} k b^{2} \delta^{2}-c_{2}\left(\alpha_{2}\right)-c_{0} \geq W_{0}
\end{aligned}
$$

(IC) make $a_{2}$,

$s_{0}+b(\pi)-\frac{1}{2} k b^{2} \delta^{2}-c_{2}\left(\alpha_{2}\right)-c_{0}$ maximize. 
First order model of IC is: $b f_{2}^{\prime}-c_{2}^{\prime}=0$.It can substitute IC condition.

Conclusion three: $b$ is the proportion gained by Content Providers in the whole industrial profit (that is the incentive given to Content Providers by the Trading Platform), if $b=0$, the effort from Content Providers $a_{2}=0$ in the cooperation.

Prove: let $b=0$, so (IC) in (3) change into: $\alpha_{2}$ maximization $s_{0^{-}} c_{0^{-}} c_{2}\left(a_{2}\right)$.

$s_{0}$ is the fix remuneration made in the earlier contract, and the way to gain utility maximization for Content Providers is to pay the least cost. Since $c_{0}$ is predictable, and can't be saved, and Content Providers will choose the least $c_{2}\left(a_{2}\right)$, that is $c_{2}\left(a_{2}\right)=0$. Because the proportional relationship between effort and $c_{2}\left(a_{2}\right)$, so the effort is $a_{2}=0$.

Conclusion four: The more shares the Content Providers owns, the more efforts they will give, while less efforts the Trading Platform will give.

Conclusion five: there are benefits conflicts between the Trading Platform and Content Providers.

Since $\frac{\partial \pi}{\partial \alpha}=0$ and $c^{\prime}=0$ exist together. The former means that the Trading Platform hopes the content providers to give more effort, the more, the better. The later indicates that Content Providers hope to give less effort. So, unless the Trading Platform designs a good enough incentive measures, or the Content Providers would not give effort as expected.

Also, the analysis of the conclusion three indicates that the increasing share of the income of Content Providers would have a major impact on the Trading Platform. The larger share of Content Providers cover, the more effort it will do; while the Trading Platform will make less effort, and this shows the conflict between two enterprises.

\section{CONCLUSION}

This article discusses the income allocation model between Trading Platform and upstream Content Providers in the content industrial chain under the condition of symmetric information and asymmetric information, and concludes the principles on the good operation of the Trading Platform.

(1) Reasonable risk-sharing principle

The Trading Platform should dominate the content chain to sign reasonable risk-sharing contract; under the condition of symmetric information, if the Trading Platform has the better risk-sharing capability, it should bear all risk. Under the condition of asymmetric information, the Trading Platform should deduce Content Providers to sign contract that can enable them to give more effort than the Pareto optimality, so can they realize the utility maximization.

(2) Balanced distribution of profits principle

By adjusting the profit proportion of the Content Providers (that is the size of $b$ ) to control the effort of Content Providers.

If the Trading Platform finds that the Content Providers are not willing to provide more qualified content recourses, we should examine whether it results from the unreasonableness of $b$, and by adjusting the size of $b$ to increase the level of effort. if the adjustment of $b$ is too big, It will do harm to the Trading Platform, so the Trading Platform should adjust the $\mathrm{b}$ appropriately to coordinate the operation of the whole platform. We could learn from allocation policy and proportion designed by the global mobile Internet telecom operators. Generally speaking, proportion of the information service fee between Content Providers and telecom operators should be in 90:10 - 60:40.

(3) Group interests optimization principle

Prevent parties in the content industry chain completing for inner industrial interest and damage the group utility.

Conflict in interest between the Content Providers and Trading Platform is the root cause of contradiction. So the Trading Platform should establish the mechanism, which could guarantee the reasonable profit and prevent both sides competing for the overall utility. After all the conflict of both side is less important than the interest. The Trading Platform should encourage cooperators work together to prosper the market, and promote the increment, rather than competing for the only stock, to maintain the steady relationship in cooperation.

(4) Negotiation principle

In general trade procedures, profit allocation can be reached through negotiation and bargaining, of which is helpful for cooperators to know each other better, and improve the satisfaction. At the same time, it also can ensure the equality and justification. Before making a final decision, both parties shall leave sufficient negotiation time, each party can propose its own original plan for profit allocation, and then reach an agreement through negotiation in the end.

\section{ACKNOWLEDGEMENTS}

This work is partly supported by National Social Science Fund of China ((No. 11BGL107) and Social Science Fund of State Administration of Press, Publication, Radio, Film and Television of the People's Republic of China (No. GD1216).

\section{REFERENCES}

[1] Peiyi Song, Zhaowen Huang. Research on Bilateral market pricing strategies for trading platform of digital media content products. 2012 International Conference on Management of eCommerce and e-Government, Beijing, 2012.10.

[2] Jean-Charles Rochet, Jean Tirole. Platform Competition in Two-Sided Markets. Journal of the European Economic Association. 2003(vol1, 4): 990-1029.

[3] Longsheng Huang, Zhisong Wu. Probability Theory and Mathematical Statistics. Zhengjiang University Press.2012. 